\title{
FAMILIES OF K-3 SURFACES
}

\author{
ALAN L. MAYER*
}

\section{§ 0. Introduction}

Let $V$ be a 2-dimensional compact complex manifold. $V$ is called a K-3 surface if: a) the irregularity $q=\operatorname{dim} H^{1}(V, \mathcal{O})$ of $V$ vanishes and b) the first Chern class $c_{1}$ of $V$ vanishes. The canonical sheaf (of holomorphic 2-forms) $K$ of such a surface is trivial, since $q=0$ implies that the Chern class map $c_{1}$ : $\operatorname{Pic}(V) \rightarrow H^{2}(V, Z)$ is injective: thus $V$ has a nowhere zero holomorphic 2-form.

Max Noether showed that the family of nonsingular quartic surfaces in $\boldsymbol{P}^{3}$ form a family of K-3 surfaces depending on 19 moduli. The collection of double coverings of $\boldsymbol{P}^{2}$ branched at a nonsingular sextic also form a family of K-3 surfaces depending on 19 moduli. Enriques and Severi (see [7]) showed how to construct, for each even integer $d>2$, a family of K-3 surfaces, depending on 19 moduli, such that 'generic' surfaces of different families are non-isomorphic. The surfaces are nonsingular surfaces of degree $d$ in $\boldsymbol{P}^{q}$, where $d=2 g-2$, generalizing the family of Noether. The construction of Enriques and Severi depends upon the technique of degeneration and the proofs are incomplete. We shall effect this construction, using the transcendental methods developed by Andreotti, Weil, and Kodaira. (A somewhat weaker result is contained in Tjurina, [14] ch. IX) The notion of "generic" is interpreted as 'with Picard number $\rho=1$ ', which phenomenon occurs everywhere outside a countable union of subvarieties.

Implicit in the work of Enriques-Severi is the claim that any algebraic K-3 surface is in some sense a specialization of a surface in one of these families (including the family of double planes).

We shall see that this is the case if specialization is taken to mean "direct deformation" in the sense of Kodaira-Spencer. We also investi-

Received November 4, 1971.

* Work partially supported by N.S.F. 
gate when birational models (with singularities) of these specialized surfaces occur on the boundary of the Chow variety of the Enriques-Severi families. We show that this occurs precisely for surfaces having the "platonic singularities" discussed by du Val (cf. [1], [2], [4]). This generalizes results of Brieskorn [3] in the $d=4$ case.

We should note that the irreducibility of the Enriques-Severi families is unsettled except in the case $d \leq 8$. However the families have certain natural "closures" (which are not compact) and the union of the closures of the families was shown to be connected by Kodaira [9] (see also Tjurina [14]). Very little is known about the boundary phenomena in general: we have some discussion of the "overlaps" of the closures of the various families which can occur.

One should remark on the analogy presented by the different families of (polarized) abelian varieties, corresponding to the different elementary divisors. In this case the different families are isogeneous, while there is no description of the relation between the families in the K-3 case. (The K-3's are more intimately connected with rational pencils of canonically embedded curves of positive genus, as we shall see.)

We shall give a "homological" criterion for ampleness and the analogue of the Lefschetz embedding theorem which points out the analogies with abelian varieties.

\section{§1. Series without fixed components}

Let $\mathscr{L}$ be an invertible sheaf on a K-3 surface $V, h^{i}(\mathscr{L})=\operatorname{dim} H^{i}(V, \mathscr{L})$, $c_{1}(\mathscr{L})=$ the Chern class of $\mathscr{L}$. The Riemann-Roch theorem gives $h^{0}(\mathscr{L})+h^{0}\left(\mathscr{L}^{-1}\right)=2+(1 / 2) c_{1}(\mathscr{L})^{2}[V]+h^{1}(\mathscr{L})$. If the complete linear series $|\mathscr{L}|$ contains a positive divisor $D>0$, then $h^{0}\left(\mathscr{L}^{-1}\right)=0$, so $l(D)$ $=2+(1 / 2) D^{2}+h^{1}(D)$, where $h^{i}(D)=h^{i}(\mathscr{L}(D)), l(D)=h^{0}(D)$ and $d=D^{2}$ $=c_{1}(\mathscr{L})^{2}[V]$, the selfintersection number, is called the degree of $D$. Since the canonical sheaf $\mathscr{K} \cong \mathcal{O}_{V}$ the arithmetic genus $g$ of $D$ is given by $d=2 g-2$. So for a positive $D$

$$
l(D)=\operatorname{dim}|D|+1=g+1+h^{1}(D)
$$

If $D$ is a curve with $k$ connected components it follows from a theorem of Kodaira ([8] Theo. 4) that $h^{1}(D)=k-1$. Thus if $C$ is an irreducible curve with $\operatorname{dim}|C|=0$ then $C$ is nonsingular rational of degree -2 . 
Such curves will be called "conics"*.

Let $\mathscr{L}$ be an invertible sheaf of degree $d=c_{1}(\mathscr{L})^{2}[V] \geq 0$. The RiemannRoch formula shows that either $\mathscr{L} \cong \mathscr{O}_{V}$ or either $|\mathscr{L}|$ or $\left|\mathscr{L}^{-1}\right|$ contains a positive divisor.

Proposition 1. Let $|\mathscr{L}|$ contain a positive divisor but have no fixed components. Then either

i) $d=$ degree of $\mathscr{L}$ is positive, $h^{1}(\mathscr{L})=0$, and $|\mathscr{L}|$ has no base points and contains an irreducible nonsingular curve $D$ of genus $g=$ $(1 / 2) d+1$, or

ii) $d=0, h^{1}(\mathscr{L})=k-1$ and $|\mathscr{L}|$ is composed of $k$ copies of an elliptic pencil, i.e., each element of $|\mathscr{L}|$ can be written as $E_{1}+\cdots+E_{k}$ with $E_{i} \in|F|, F$ being nonsingular elliptic.

Proof. a) Assume $|\mathscr{L}|$ contains an irreducible $D$. If furthermore $d$ is positive then the assertions of i) are proved in [14] ch. VIII sec. $3^{* *}$. If $d$ is $\leq 0$ then $D$ positive and $h^{1}(D)=0$ implies $d=-2$ or 0 . But if $d=-2, D$ is a conic and a fixed component of $|\mathscr{L}|$, and if $d=0$ then $|\mathscr{L}|$ has no base points, so by Bertini's second theorem a generic $D$ is nonsingular, and since $d=0$, elliptic.

b) If $|\mathscr{L}|$ does not contain an irreducible divisor, by Bertini's first theorem $|\mathscr{L}|$ is composed of a pencil. If the pencil in question were irrational it would have no base points (Zariski, Algebraic Surfaces p. 25), so its members would have zero selfintersection and thus give a fibre space of elliptic curves. But an elliptic fibre space whose total space is a K-3 must have a rational base. For if $E$ is a general fibre and $E \neq E^{\prime} \in|E|$, then $E^{\prime}$. (any fibre) $=0$, but since $E^{\prime}$ must intersect some fibre, it must coincide with a fibre. This gives a rational map of

* This is perhaps a misleading word e.g. in [5] actual conics occur, but we shall use it for the moment.

** Note: The proof in [14] that $|D|$ has no base points and a generic fibre is nonsingular is vague, as it uses the Bertini theorem which is proved only for very ample linear series. However the desired result may be proved as follows: the proof of Lemma 2 p. 187 of [14] shows that no base points can be singular points of $D$. It follows that the base points of a generic pencil containing $D$ are nonsingular points of $D$ blowing up these points we obtain a morphism of the blown up surface onto $\boldsymbol{P}^{\mathbf{1}}$. Its generic fibre is nonsingular (Sard's theorem) but a generic fibre is the proper transform of a generic curve of $D$ and isomorphic to it, since the singular locus of this curve may be assume disjoint from the base locus. Thus a generic element of $D$ is nonsingular. It follows that $|D|$ cuts out the canonical series on the curve, and since this has no base points, the series $|D|$ has no base points. 
the series $|E|$ onto the base space, so by Lueroth's theorem, the base is rational. Thus the pencil in question is rational. If $F$ is a generic member of the pencil we must have $\operatorname{dim}|F|=1$, otherwise there would be an $F^{\prime}$ in $|F|$ not in the pencil, and this would contradict the completeness of $|\mathscr{L}|$. Since $h^{1}(F)=0$ we have $F^{2}=0$, so $F$ is elliptic. Thus the elements of $|\mathscr{L}|$ have the form $D=E_{1}+\cdots+E_{k}$ with $E_{i}$ in $|F|$ : since $E_{i} \cdot E_{j}=0$ and the $E_{i}$ are in general distinct, the generic $D$ is a curve with $k$ connected components, and so $h^{1}(\mathscr{L})=k-1$. We see that $d=D^{2}=0$ and $\operatorname{dim}|\mathscr{L}|=k$. Note that the elliptic pencil has no base points

We shall examine the case i) in more detail.

Proposition 2. Let $|\mathscr{L}|$ have no fixed components and contain a positive divisor of degree $d>0$. Then either

ia) $|\mathscr{L}|$ contains an irreducible nonsingular nonhyperelliptic curve $D$ of genus $g=(1 / 2) d+1$ and the morphism $\varphi_{\mathscr{L}}: V \rightarrow P\left(H^{0}(V, \mathscr{L})\right)$ defined by the sheaf $\mathscr{L}$ is a birational map of $V$ onto a projectively normal surface of degree $d$ whose only singularities are rational double points. The fibres over these double points are one of the five types of configurations of du Val: cf. Figure 2.8 in Artin [1].

or ib) The general curve $D$ is hyperelliptic of genus $g$ and $\varphi_{\mathscr{S}}$ is a rational map of degree 2 of $V$ onto a normal rational surface of degree $g-1$. However $\varphi_{\mathscr{L} 3}$ is a birational morphism, and so is $\varphi_{\mathscr{L}^{2}}$ unless $g=2$.

Proof. Since $\mathscr{K}_{V}$ is trivial the adjunction formula gives $\mathscr{L} \otimes \mathcal{O}_{D}=$ $\mathscr{K}_{D}$ the canonical sheaf on $D$, a nonsingular member of $|\mathscr{L}|$. The exact sequence $0 \rightarrow \mathcal{O}_{V} \rightarrow \mathscr{L} \rightarrow \mathscr{L} \otimes \mathcal{O}_{D} \cong \mathscr{K}_{D} \rightarrow 0$ with the fact that $H^{1}\left(V, \mathcal{O}_{V}\right)$ $=0$ shows that $|\mathscr{L}|$ cuts out the complete canonical series $\left|\mathscr{K}_{D}\right|$ on $D$. More generally tensoring the above sequence with $\mathscr{L}^{n}$ and observing that $H^{1}\left(V, \mathscr{L}^{n}\right)=0$ (by Prop. 1) for all positive $n$, we have exact sequences $0 \rightarrow \mathscr{L}^{n} \rightarrow \mathscr{L}^{n+1} \rightarrow \mathscr{K}_{D}^{n+1} \rightarrow 0$, and $\mathscr{L}^{n+1}$ cuts out the $(n+1)$-pluricanonical series on $D$. Since $\mathscr{L}^{n}$ has no base points we have a morphism $\varphi_{\mathscr{L}^{n}}: V$ $\rightarrow \boldsymbol{P}\left(H^{0}\left(V, \mathscr{L}^{n}\right)\right)$ for all positive $n$. Now $\mathscr{K}_{D}$ is very ample in the nonhyperelliptic case so $\varphi_{\mathscr{L}} \mid D$ is birational: this holds at almost all fibres $D$ of $|\mathscr{L}|$ so $\varphi_{\mathscr{L}}$ is generically 1-1 and so birational. In the hyperelliptic case $\mathscr{K}_{D}^{3}$ is very ample (or $\mathscr{K}_{D}^{2}$ if $g \neq 2$ ) so by the same argument the morphisms $\varphi_{\mathscr{L}^{3}}$ (or $\varphi_{\mathscr{L}^{2}}$ if $g \neq 2$ ) are birational. In the hyperelliptic case 
$\left|\mathscr{K}_{D}\right|$ is compounded of the hyperelliptic involution, and $\varphi_{\mathscr{K}_{D}}$ is a $2-1$ map onto a twisted rational curve: thus $\varphi_{\mathscr{L}}$ is generically a 2-1 map onto a rational surface. The image $\bar{V}$ of $\varphi_{\mathscr{L}}$ clearly has degree $d / 2$ or $d$ according to whether or not $D$ is hyperelliptic. We shall not deal with the structure of $\bar{V}$ in the hyperelliptic case. To show that $\bar{V}$ is projectively normal in the nonhyperelliptic case, we must show that $H^{0}(V, \mathscr{L})$ generates the graded ring $\bigoplus_{n \geq 1} H^{0}\left(V, \mathscr{L}^{n}\right)^{*}$. One proceeds by induction on $n$, the case $n=1$ being clear. Let $f_{0}, \cdots, f_{g}$ span $H^{\circ}(V, \mathscr{L})$ where $f_{0}$ is the section whose vanishing defines $D$. Then the restrictions $\bar{f}_{1}, \cdots, \bar{f}_{g}$ of the other $f_{i}$ to $D$ span $H^{\circ}\left(D, \mathscr{K}_{D}\right)$, and by the theorem of M. Noether (see e.g. Severi's Vorlesungen über algebraische Geometrie) they generate the graded ring $\underset{n \geq 1}{\oplus} H^{0}\left(D, \mathscr{K}_{D}^{n}\right)$. Now let $f$ be an arbitrary element of $H^{0}\left(V, \mathscr{L}^{n+1}\right)$, and let $\bar{f}$ be its restriction to $D$ : so $\bar{f}=P\left(\bar{f}_{1}, \cdots, \bar{f}_{g}\right)$ where $P$ is a homogeneous polynomial of degree $n+1$. The section $f-$ $P\left(f_{1}, \cdots, f_{g}\right)$ then vanishes on $D$, so by the abovementioned exact cohomology sequence it may be expressed as $f_{0} h$, with $h$ in $H^{0}\left(V, \mathscr{L}^{n}\right)$. But by the induction hypothesis $h=Q\left(f_{0}, \cdots, f_{g}\right)$ where $Q$ is a homogeneous polynomial of degree $n$. Thus $f=P\left(f_{1}, \cdots, f_{g}\right)-f_{0} Q\left(f_{0}, \cdots, f_{g}\right)$ so the $f_{0}, \cdots, f_{g}$ are seen so generate all of $\bigoplus_{n \geq 1} H^{0}\left(V, \mathscr{L}^{n}\right)$. (A similar argument shows $\bar{V}$ normal in the hyperelliptic case). Thus $\bar{V}$, in particular, has at most isolated singularities. If $C_{i}$ are the irreducible components of $\varphi_{\mathscr{S}}^{-1}(P)$, for $P$ any singular points, the intersection number matrix $\left\|\left(C_{i} C_{j}\right)\right\|$ is negative definite by a theorem of Mumford [12]. In particular $C_{i}^{2}<0$ for all $i$ and by the Riemann-Roch formula this means the $C_{i}^{2}=-2$ i.e. the $C_{i}$ are conics. Thus $\varphi_{\mathscr{S}}^{-1}(P)$ (see Artin [1] Fig. 2.8) is one of the five possible types of 'platonic configurations' of du Val, that is, $P$ is a rational double point. (Artin [1], Brieskorn [4])

\section{§ 2. Generic Surfaces}

Let $\rho(V)=\operatorname{rank}\left(\operatorname{Pic}(V)=H^{1}\left(V, \mathcal{O}_{V}^{*}\right)\right)$ denote the Picard number of a K-3 surface $V$. Let us call $V$ a generic surface of type $d$ if $\rho(V)=1$ and if Pic $(V)$ is generated by a sheaf $\mathscr{L}$ of degree $d$ : i.e. $c_{1}(\mathscr{L})^{2}[V]=d$. Note that $d$ is always even (see [14] ch. IX)

* For by Zariski's projective normalization [17] the normalization of $\bar{V}$ is projectively embedded by a multiple of the hyperplane section, hence the normal model is embedded by the sheaf $\mathscr{L}^{n}$ for some $n$. But since the sections of $\mathscr{L}^{n}$ come from sections of $\mathscr{L}$, it follows that $\bar{V}$ is already normal. (I am indebted to T. Matsusaka for this argument). 
Proposition 3. Let $V$ be a generic surface of type $d$. Then

a) if $d<-2, V$ contains no curves.

b) if $d=-2, V$ contains a unique conic, and has no nonconstant meromorphic functions.

c) if $d=0, V$ is a nonalgebraic elliptic fibre space over a rational curve, whose only singular fibres are rational curves with either a node or a cusp.

d) if $d=2, V$ is a double cover of $\boldsymbol{P}^{2}$ branched at an irreducible sextic.

e) if $d>2$, then $V$ is a nonsingular surface of degree $d=2 g-2$ in $\boldsymbol{P}^{g}$ whose generic hyperplane section is a canonically embedded nonhyperelliptic curve of genus $g$.

Proof. a), b) By the Riemann-Roch formula an irreducible curve has degree $\geq-2$, and equality occurs only for a conic, and by the same argument if $d=-2$, either $\operatorname{dim}|\mathscr{L}|$ or $\operatorname{dim}\left|\mathscr{L}^{-1}\right|$ is $\geq 0$ and so $V$ contains an irreducible curve, which is seen to be a conic $C$ which is unique on $V$ since $\operatorname{dim}|C|=0$.

c) If $d=0$ replacing $\mathscr{L}$ by $\mathscr{L}^{-1}$ if necessary, one sees that the elements of $|\mathscr{L}|$ form an elliptic pencil without base points, that is an elliptic fibre space over $\boldsymbol{P}^{1}$. Since $\rho(V)=1$, no multiple or reducible fibres can occur, so the fibres are either nonsingular or rational curves with a node or a cusp (by [9]). Since an ample sheaf has positive degree $V$ is nonalgebraic.

d) If $d=2$ then $|\mathscr{L}|$ (or, again $\left|\mathscr{L}^{-1}\right|$ ) contains a nonsingular curve $D$ of genus 2 , and since $\rho(V)=1$, the fundamental locus of $\varphi_{\mathscr{S}}$ is empty (for its components are disjoint from $D$ ), so $\varphi_{\mathscr{L}}$ is a double covering of $\boldsymbol{P}^{2}$. If $B$ is the branch curve, then $B$ intersects $D$ in the 6 distinct Weierstrass points of $D$, and $B \in|3 D|$. If $B=B^{\prime}+B^{\prime \prime}$ is reducible, then (say) $B^{\prime} \in|D|$, so $B^{\prime} \cap D=P_{1}+P_{2}$ is a canonical divisor on $D$, where $P_{1}$ and $P_{2}$ are distinct Weierstrass points. But this is impossible since $2 P_{1}$ is a canonical divisor.

e) If $d>2$, then $|\mathscr{L}|$ (or $\left|\mathscr{L}^{-1}\right|$ ) contains a nonsingular curve $D$ : we shall show that $D$ is nonhyperelliptic, then the desired result follows from Proposition 1, since $\rho(V)=1$ implies that the $\mathscr{L}$ ample, so $\varphi_{\mathscr{L}}$ is an isomorphism. In the case $d=4$, if $D$ is hyperelliptic $\bar{V}=\varphi_{\mathscr{L}}(V)$ is a quadric surface in $\boldsymbol{P}^{3}$. If $H$ is a hyperplane in $\boldsymbol{P}^{3}$ such that $H \cap \bar{V}=2$ 
lines, then the pullback of $H$ to $V$ is a reducible element of $|\mathscr{L}|$ and so $\mathscr{L}$ cannot generate $\operatorname{Pic}(V)$. For $d>4$, if $D$ were hyperelliptic $\varphi_{\mathscr{L}}$ would be a double covering branched at a curve $B$, with $B \cdot D=2 g+2$, since $\varphi_{\mathscr{K}_{D}}$ is ramified at the $2 g+2$ Weierstrass points of $D$. Since $\mathscr{L}$ generate Pic $(V)$ we must have $B \sim m D$ for some positive integer $m$, so $2 g+2=$ $m(2 g-2)$. Clearly $m \geq 2$ so $g+1 \geq 2 g-2$ which is impossible since $g>3$ if $d>4$. Thus $D$ cannot be hyperelliptic.

\section{§3. Existence of $\mathbf{K}-3 \mathrm{~s}$.}

In this section we shall give a sort of converse to case ia) of Proposition 2. Let $\bar{V}$ be an irreducible surface of degree $d=2 g-2$ in $P^{g}$, with $g \geq 3$. $\bar{V}$ will be called a "canonical surface" if

a) $\bar{V}$ has at most rational double points as singularities

b) if $H$ is a generic hyperplane in $\boldsymbol{P}^{g}$ then $D=H \cdot \bar{V}$ is a nonsingular curve of genus $g$ canonically embedded in $H$. (This merely means that $\bar{V}$ lies in no hyperplane of $\boldsymbol{P}^{g}$.)

Proposition 4. Let $\bar{V} \subset P^{g}$ be a canonical surface. Then there is a K-3 surface $V$ with an invertible sheaf $\mathscr{L}$ such that $\varphi_{\mathscr{L}}(V)=\bar{V}$, with $\varphi_{\mathscr{L}}^{-1}\left(\mathcal{O}_{\bar{V}}(1)\right)=\mathscr{L}$.

Proof. Let $H$ be a generic hyperplane in $\boldsymbol{P}^{g}$ so that $D=H \cdot \bar{V}$ is a nonsingular curve on $\bar{V}$, disjoint from the singular locus. Let $\pi: V$ $\rightarrow \bar{V}$ be an admissible resolution of singularities of $\bar{V}$, in the sense of Brieskorn [4], so that $R^{1} \pi_{*}\left(V, \mathcal{O}_{\bar{V}}\right)=0$, and the resolving fibres contain no exceptional curves of the first kind. Let $\mathscr{L}$ be the pullback of the sheaf $\mathcal{O}_{\bar{v}}(1)$ by $\pi$. For each positive integer $n$ we have an exact sequence

$$
0 \rightarrow \mathcal{O}_{\bar{V}}(n-1) \rightarrow \mathcal{O}_{\bar{V}}(n) \rightarrow \mathscr{K}_{D}^{n} \rightarrow 0
$$

Note that $h^{0}\left(\mathcal{O}_{\bar{V}}(1)\right)=g+1$ (since $\bar{V}$ cannot lie in a hyperplane, otherwise $D$ would not span the generic $H)$ and $h^{0}\left(\mathscr{K}_{D}\right)=g, h^{1}\left(\mathscr{K}_{D}\right)=1$, and $h^{1}\left(\mathscr{K}_{D}^{n}\right)$ $=0$ for $n>1$. Thus the exact cohomology sequence of $(* 1)$ is

$$
\begin{aligned}
0 & \rightarrow H^{0}\left(\bar{V}, \mathcal{O}_{\bar{V}}\right) \rightarrow H^{0}\left(\bar{V}, \mathcal{O}_{\bar{V}}(1)\right) \rightarrow H^{0}\left(D, \mathscr{K}_{D}\right) \rightarrow H^{1}\left(\bar{V}, \mathcal{O}_{\bar{V}}\right) \\
& \rightarrow H^{1}\left(\bar{V}, \mathcal{O}_{\bar{V}}(1)\right) \rightarrow C \rightarrow H^{2}\left(\bar{V}, \mathcal{O}_{\bar{V}}\right) \rightarrow H^{2}\left(\bar{V}, \mathcal{O}_{\bar{V}}(1)\right) \rightarrow 0 .
\end{aligned}
$$

This shows that $H^{1}\left(\bar{V}, \mathcal{O}_{\bar{V}}\right) \subset H^{1}\left(\bar{V}, \mathcal{O}_{\bar{V}}(1)\right)$. In the same way the cohomology sequence of $(* n)$ for all $n$ shows 


$$
\begin{aligned}
H^{1}\left(\bar{V}, \mathcal{O}_{\bar{V}}\right) \subset H^{1}\left(\bar{V}, \mathcal{O}_{\bar{V}}(1)\right) \subset H^{1}\left(\bar{V}, \mathcal{O}_{\bar{V}}(2)\right) \subset \cdots \\
H^{2}\left(\bar{V}, \mathcal{O}_{\bar{V}}(1)\right) \subset H^{2}\left(\bar{V}, \mathcal{O}_{\bar{V}}(2)\right) \subset \cdots
\end{aligned}
$$

and so by "theorem $B$ " (cf. [16]) $h^{1}\left(\mathcal{O}_{\bar{V}}\right)=0$ and $h^{2}\left(\mathcal{O}_{\bar{V}}(1)\right)=0$. Applying the latter to cohomology of $\left({ }^{*} 1\right)$ we obtain $h^{2}\left(\mathcal{O}_{\bar{V}}\right)=1$, and so the Euler characteristic $\chi\left(\bar{V}, \mathcal{O}_{\bar{V}}\right)=2$. It follows from the Leray spectral sequence and the fact that $R^{1}\left(\pi_{*}\left(V, \mathcal{O}_{V}\right)=0\right.$ that $\chi\left(V, \mathcal{O}_{V}\right)$ also $=2$ (cf. Artin [1] Theo. 2.7). So by the duality theorem $h^{0}\left(\mathscr{K}_{V}\right)=h^{2}\left(\mathcal{O}_{V}\right)=h^{1}\left(\mathcal{O}_{V}\right)$ +1 , where $\mathscr{K}_{V}$ is the canonical sheaf on $V$. Identifying $D$ with $\pi^{-1}(D)$ on $V, \mathscr{L}=\mathcal{O}(D)$ and we have the exact sequences

$$
0 \rightarrow \mathscr{L}^{n-1} \rightarrow \mathscr{L}^{n} \rightarrow \mathscr{K}_{D}^{n} \rightarrow 0
$$

for all positive integers $n$. Now $h^{2}(\mathscr{L})=h^{0}\left(\mathscr{K}_{V} \otimes \mathscr{L}^{-1}\right)$ must vanish. For if $F \in\left|\mathscr{K}_{V} \otimes \mathscr{L}^{-1}\right|$ then $F+D \in\left|\mathscr{K}_{V}\right|$. But since $\mathscr{K}_{D}=\left(\mathscr{L} \otimes \mathscr{K}_{V}\right) \otimes$ $\mathcal{O}_{D}$ (by the adjunction formula) $\mathscr{K}_{V} \otimes \mathcal{O}_{D}$ is trivial, so $D \cdot(F+D)=0$, i.e. $D \cdot F=-d<0$. But this is impossible since $F$ is $\geq 0, D$ irreducible and $d=D^{2}>0$. Thus the exact cohomology sequence of $(* * 1)$ gives

$$
0 \rightarrow H^{1}\left(V, \mathcal{O}_{V}\right) \rightarrow H^{1}(V, \mathscr{L}) \rightarrow C \rightarrow H^{2}\left(V, \mathcal{O}_{V}\right) \rightarrow 0
$$

This sequence gives $h^{1}(\mathscr{L})+1=h^{2}\left(\mathcal{O}_{V}\right)-h^{1}\left(\mathcal{O}_{V}\right)$ which $=1$. Thus $h^{1}(\mathscr{L})$ $=0$ and so by the same sequence $h^{1}\left(\mathcal{O}_{V}\right)=0$, and so $h^{0}\left(\mathscr{K}_{V}\right)=1$. It follows that $h^{0}\left(\mathscr{K}_{V}^{n}\right)$ are $\geq 1$ for all positive $n$. But since $\mathscr{K}_{V} \otimes \mathcal{O}_{D}$ is trivial, any element of $\left|n \mathscr{K}_{V}\right|$ is disjoint from $D$, and hence lies on the fundamental locus of $\pi$. Thus $\operatorname{dim}|n \mathscr{K}|=0$ for all $n$. Sincely has arithmetic genus and all plurigenera $=1$, by the classification theorem of Enriques (see [14]) $V$ is the result of applying (perhaps) some modifications on a K-3 surface. But if $V$ contains exceptional curves, they are part of a canonical divisor, and hence do not intersect $D$. Thus any exceptional curves must lie on the fundamental locus of $\pi$, which is ruled out by our construction. Thus $V$ is itself in fact a $\mathrm{K}-3$ surface, and $\mathscr{L}$ an invertible sheaf of the type described in Proposition 2, case ia). We have a morphism from $\bar{V}$ to $\boldsymbol{P}\left(H^{0}(V, \mathscr{L})\right)$ since $H^{0}(V, \mathscr{L})=H^{0}\left(\bar{V}, \mathcal{O}_{\bar{V}}(1)\right)$ and $\left|\mathcal{O}_{\bar{V}}(1)\right|$ has no base points. This gives a birational morphism of $\bar{V}$ onto $\varphi_{\mathscr{L}}(V)$, and since if $P \neq Q$ on $\bar{V}$ there is a hyperplane through $P$ but not $Q$, this morphism is injective, and so by Zariski's main theorem, an isomorphism.

Remark. Note the $\operatorname{dim} H^{0}\left(\boldsymbol{P}^{q}, \mathcal{O}_{\boldsymbol{p}}(h)\right)=\left(\begin{array}{c}g+h \\ h\end{array}\right)$ while, since we see 
that $|h D|$ has no fixed components $l(h D)=2+h^{2}(g-1)$ by the RiemannRoch theorem. It follows that $\bar{V}$ lies in the intersection of precisely $(g-2)(g-3) / 2$ linearly independent quadric hypersurfaces. In the case $g=4$ we see that there are $15=35-20$ linearly independent cubics through $V$, and precisely one quadric. Thus $\bar{V}$ is the intersection of a cubic and a quadric. It is an elementary excercise to see that the intersection of a generic cubic and quadric is a K-3 surface in $\boldsymbol{P}^{4}$ : thus we see that the collection of all our $\bar{V}$ 's in $\boldsymbol{P}^{4}$ form an open set on an irreducible component of the Chow variety. In the case $d=8, g=5 \bar{V}$ is contained in 3 linearly independent quadrics, and their intersection, being of degree 8 , coincides with $\bar{V}^{*}$. Conversely the intersection of 3 generic quadrics in $\boldsymbol{P}^{5}$ is easily seen to be a K-3 surface, so again our family is irreducible. Since $2^{g-2}>2 g-2$ except if $g \leq 5$, it is clear that for $g>5$ our $\bar{V}$ cannot be the complete intersection of $g-2$ hypersurfaces. On the other hand one might conjecture that $\bar{V}$, in general, is an intersection of a large number of quadric hypersurfaces, in analogy with the theorem of Enriques-Petri for the case of canonical curves. Incidentally, the above shows that the generic curve of genus $\leq 5$ (the cases $g=1$ and $g=2$ deserve special treatment) appears as a curve on a K-3 surface. However a heuritic count of moduli shows that the curves in $|\mathscr{L}|$ for generic $\bar{V}$ exhaust an open part of the modular variety of curves only if $g+19 \geq 3 g-3$, i.e. for $g \leq 8$. (Whether this in fact happens for $g=6,7$, does not seem to be known.) Nevertheless one might conjecture** for any $g$ the family of $\bar{V}$ 's is irreducible (cf. Enriques [7]), and the suggestions of B. d'Orgeval [5]).

\section{$\S 4$. Fixed components and ampleness}

Let $\mathscr{L}$ be an invertible sheaf of degree $d>0$ on a K-3 surface $V$. Then (replacing $\mathscr{L}$ with $\mathscr{L}^{-1}$ if necessary) $|\mathscr{L}|$ contains a positive divisor $D$ and the union $F$ of all its fixed components is a union of its connected components $F_{i}$. Since $\operatorname{dim}\left|F_{i}\right|=0$, each $F_{i}$ is a union of conics, $F_{i}^{2}=$ -2 , and the $F_{i}$ 's have the 'platonic' structures described [1]. The fixed divisor $D_{f}$ of $|\mathscr{L}|$ has support $F$, and the residual linear series $|\mathscr{L}|=$ $\left|D-D_{f}\right|$ contains a positive divisor $D^{\prime}$ with $D^{\prime 2}=d^{\prime} \geq 0$. By Proposition 1 either $D^{\prime}$ can be assumed nonsingular of genus $g^{\prime}=d^{\prime} / 2+1>1$

* $\bar{V}$ is reduced so we do not get the double Veronese surface.

** This follows from the recent work of (Šafarevič and Piatetskii-Šapiro: Izvestia Akad. Nauk. 1971). 
or $D^{\prime}$ is a disjoint union of $k$ nonsingular elliptic curves $E_{j}$ of an elliptic pencil.

We shall call a connected component $F_{i}$ a type I component if $F_{i}$ is disjoint from $D^{\prime}$ : otherwise, we shall call $F_{i}$ a type II component. For any $F_{i}$, since $D^{\prime} \leq D^{\prime}+F_{i} \leq D$, we have $l\left(D^{\prime}\right)=l\left(D^{\prime}+F_{i}\right)=\mathscr{X}(D)$. If $D^{\prime}$ is irreducible and $F_{i}$ is type II then $D^{\prime}+F_{i}$ is connected, so $h^{1}\left(D^{\prime}+F_{i}\right)=h^{1}\left(D^{\prime}\right)=0$. Thus by the Riemann-Roch formula, $D^{\prime 2}=$ $\left(D^{\prime}+F_{i}\right)^{2}$ so $D^{\prime} F_{i}=1$. If on the other hand $D^{\prime}$ is a union of elliptic curves $E_{1}, \cdots, E_{k}$ then $h^{1}\left(D^{\prime}\right)=k-1$, and if $F_{i}$ is of type II it intersects all the $E_{j}$ so again $D^{\prime}+F_{i}$ is connected and $h^{1}\left(D^{\prime}+F_{i}\right)=0$. The Riemann-Roch formula then gives $k-1=\left(D^{\prime}+F_{i}\right)^{2} / 2$ or $k=D^{\prime} \cdot F_{i}=$ $\sum_{j} E_{j} \cdot F_{i}$, so that $E_{j} \cdot F_{i}=1$ for all $j$. Note that in this case $|\mathscr{L}|$ must contain at least one type II component, for otherwise we would have $D^{2}=\left(D^{\prime}+D_{f}\right)^{2}=D_{f}^{2} \leq 0$. Let $F_{0}$ be any such component. Now let us define $D_{0}$ to be $D^{\prime}$ in the case where $D^{\prime}$ is irreducible and to be $D^{\prime}+F_{0}$ in the case where $D^{\prime}$ is a union of $k$ elliptic curves. Then $d_{0}=D_{0}^{2}=d^{\prime}$ in the first case and $d_{0}=2 k-2$ in the second case. Then $D_{0}$ has arithmetic genus $g_{0}=d_{0} / 2+1$, which is in any case half the first Betti number of $D_{0}$ (in the elliptic case $g_{0}=1$ ). Note that $\operatorname{dim}|\mathscr{L}|=\operatorname{dim}\left|D_{0}\right|$ $=g_{0}$ in all cases. Note that in the elliptic case $F_{0}$ is uniquely determined: for if $F_{1}$ were another component of type II then $F_{1} \cdot D_{0} \geq F_{1} \cdot D^{\prime}=k$ and since $D_{0}+F_{1} \leq D, \operatorname{dim}\left|D_{0}+F_{1}\right|=k$ : but $D_{0}+F_{1}$ would be connected so the Riemann-Roch theorem would give $\left(D_{0}+F_{1}\right)^{2} \geq 4 k-4$ equal to $2 k-2$. Note also that $F_{0}$ will consist of a conic meeting the $E_{j}$ plus some bad components of the elliptic fibration, which do not intersect the general elliptic curve. Collecting this:

Proposition 5. Let $D$ be a positive divisor with $d=D^{2}>0$, then there is a connected curve $D_{0} \leq D$ which is either irreducible or consists of $g_{0}$ elliptic curves in an elliptic pencil joined by a platonic rational curve $F_{0}$ meeting each elliptic curve once. The series $\left|D_{0}\right|$ has no fixed points, except, in the elliptic case, those on the rational curve, and $D_{0}^{2}=2 g_{0}-2, g_{0}=\operatorname{dim}\left|D_{0}\right|=\operatorname{dim}|D| . \quad$ Any divisor in $|D|$ consists of $a$ divisor in $\left|D_{0}\right|$ plus some fixed components. If $g$ is the arithmetic genus of $D$ then $g=g_{0}-h^{1}(D)$. If $F_{i}$ is any connected component of the locus of fixed components of $|D|$ other than $F_{0}$, then either $F_{i} \cdot D_{0}=0$ (type I) or $F_{i} \cdot D_{0}=1$ (type II). 
COROLlary. If $D$ is divisor with $D^{2}>0$ then the linear series $|3 D|$ is gives a birational morphism of $V$.

Proof. It suffices to show that $\left|3 D_{0}\right|$ gives a birational morphism where $D_{0}$ is an above. In the case where $D_{0}$ is irreducible this follows from Proposition 2. In the case where $D_{0}=F_{0}+E_{1}+\cdots+E_{k}$ the variable part of the linear series has no base points and no two elliptic curves of the fibration are identified under the morphism. On the other hand on a general elliptic curve $\left|3 D_{0}\right|$ cuts out a linear series of degree 3 some generic elliptic $E$, and if this is complete, it will be very ample on $E$ and the map, being generically biregular, will be birational. To show that the linear series is in fact complete, it suffices by the usual exact sequence to show that $h^{1}\left(3 D_{0}-E\right)=0$. Now $\left(3 D_{0}-E\right)^{2} \geq 9 d_{0}-$ $6>0$ and since $3 D_{0}-E \geq D_{0}$, the only fixed component $3 D_{0}-E$ could have would be $F_{0}$, Now since $3 D_{0}-E \geq 2 D_{0}$ it suffices to show that $F_{0}$ is not a fixed component of $2 D_{0}$. It could only occur as a fixed component with multiplicity one or two. Since $2 D_{0}-F_{0}=2 g_{0} E+F_{0}$ has a fixed component, it would have to occur with multiplicity two. But then $l\left(2 D_{0}\right)=l\left(2 D_{0}-2 F_{0}\right)=2+2 g_{0}-1$ while we have $l\left(2 D_{0}\right) \geq 2+\left(2 D_{0}\right)^{2} / 2$ $=2+2 d_{0}=4 g_{0}-2$. But $2 g_{0}+1 \geq 4 g_{0}-2$ only if $g_{0}=1$, i.e. $d_{0}=0$, contradicting our assumption $d>0$. Now assume that a positive divisor $D$ of degree $d>0$ is ample (in the sense of Grothendieck) i.e. for some $N$ the series $|N D|$ defines a projective embedding and has no fixed components (is very ample). By Kodaira's vanishing theorem $h^{1}(D)=0$ and so by Proposition 5, $D^{2}=D_{0}^{2}$. By Nakai's criterion [13], $D$ must have positive intersection with any curve on $V$, so $D$ can have at most fixed components of type II. Writing $D=D_{0}+D_{\text {II }}$ we have $D_{\text {II }}^{2}+2 D_{0} D_{\text {II }}=0$ and since $D_{0}$ and $D_{\text {II }}$ are positive $D \cdot D_{\text {II }}=D_{0} D_{\text {II }}+D_{\text {II }}^{2} \leq 0$, thus there can be no fixed components, except in the elliptic case. In the latter case $F_{0}$ has one irreducible component $C_{1}$ which meets the $E_{j}$. If we write $D=D_{0}+\sum n_{i} C_{i}$ where the $C_{i}$ are the irreducible components of $F_{0}$ the same argument gives all $n_{i}=0$ : Thus in the elliptic case $F_{0}$ is an irreducible conic and $D=D_{0}$. Thus we have

Proposition 6. If $D$ is ample then $D=D_{0}$.

COROLlaRY. If $D$ is ample then $3 D$ is very ample.

Proof. By the corollary to Proposition $53 D=3 D_{0}$ defines a birational morphism. No curve $C$ can collapse otherwise we would have 
$C \cdot D=0$, so this gives a projective embedding. If $D_{0}$ is irreducible then $|3 D|$ has no fixed components since $|D|$ itself has none. It remains to show that, for $D=F_{0}+E_{1}+\cdots+E_{k}, F_{0}$ is not a fixed component of $|3 D|$, which follows a fortiori from the proof of the Corollary to Proposition 5. (Note the analogy this presents with Lefschetz's embedding theorem for abelian varieties.)

Remark. One can construct a series $|D|$ with fixed components of either type. For the $F_{0}$ in the elliptic case it suffices to take a section of the elliptic fibre space. In the other cases, if $D_{0}$ is connected with $D_{0}^{2}>0$ and $C$ is a curve collapsed under the morphism $\varphi_{D_{0}}$ then the Riemann-Roch theorem shows that $l\left(D_{0}\right)=l\left(D_{0}+C\right)$, (since $h^{1}\left(D_{0}+C\right.$ ) $=h^{1}\left(D_{0}\right)+1=1$ and $\left.\left(D_{0}\right)^{2}=\left(D_{0}+C\right)^{2}+2\right)$ and $C$ is a type I fixed component of $\left|D_{0}+C\right|$. On the other hand let $D_{0}$, which may be ample, be linearly equivalent to a divisor of the form $D_{1}+C$, with $C$ a conic and $D_{1}^{2}=D_{0}^{2}-4$ (so $D_{1} \cdot C=3$ ) : for example we can have $D_{0}$ a plane section of a nonsingular quadric in $\boldsymbol{P}^{3}$ which degenerates into the union of a line and a planar cubic, jointed at 3 points. Let $D=D_{0}+C$ : Then $C \cdot D=1, D^{2}=D_{0}^{2}$ and the fact that $D$ and $D_{0}$ are connected shows that $l(D)=l\left(D_{0}\right)$ so $C$ is a fixed component of type II of $|D|$. In general these components seem more mysterious than the type $I$.

\section{§5. Numerical criteria}

We assume that $V$ is an algebraic K-3 surface. Intersection product induces a quadratic form $\langle$,$\rangle on \operatorname{Pic}(V)$ which may be thought of as an additive subgroup of $H^{2}(V, Z)$. A class $c$ in Pic $(V)$ is called ample if it is the class of an ample divisor. Let $c$ be some given ample divisor class. Then let $K^{+}=$the set of $a \neq 0$ in Pic $(V)$ such that $\langle a, a\rangle$ $\geq-2$, and $\langle c, a\rangle\rangle 0$. If $\langle a, a\rangle \geq-2, a \neq 0$, either a or $-a$ is the class of a positive divisor, so either $\langle a, c\rangle$ or $\langle-a, c\rangle$ is positive. Thus the definition of $K^{+}$is independent of the choice of the ample class $c$, and furthermore, the class of any irreducible curve lies in $K^{+}$.

Proposition 7. A class $c$ in Pic $(V)$ is ample if and only if $\langle c, c\rangle$ $>0$ and $\langle c, a\rangle>0$ for all $a$ in $K^{+}$.

Proof. One part follows from the definition of $K^{+}$. Conversely, if $\langle c, a\rangle>0$ for all $a$ in $K^{+}$then $c$ is the class of a divisor having positive 
intersection with every irreducible curve, and since the divisor has positive selfintersection, it is ample by the criterion of Nakai [13].

Remark. A purely numerical characterization of $K^{+}$(rather than $\pm K^{+}$) cannot be given. For the numerical equivalence ring of $V$ is canonically isomorphic to the numerical equivalence ring of the complex conjugate surface to $V$ (also an algebraic $\mathrm{K}-3$ ) and considered as such, the new ample divisors are the negatives of the old ample ones. We shall call a class $b$ pseudoample if $\langle b, b\rangle\rangle 0$ and $\langle b, a\rangle \geq 0$ for all $a$ in $K^{+}$. (cf. S. Kleiman, Thesis Harvard 1964)

Proposition 8. A class $b$ is pseudoample if and only if it is the class of a divisor $D$ with $D^{2}>0$ such that $|D|$ has no fixed components, except perhaps the fixed component $F_{0}$, in the case where the free part of $D$ is an elliptic pencil. (in the terminology of Proposition 5 this means $\left.|D|=\left|D_{0}\right|\right)$

Proof. Assume that $b$ is the class of $D$ with $D=D_{0}$. Then $|3 D|$ gives a birational morphism of $V$ and has no fixed components: hence if $C$ is any irreducible curve $C \cdot D>0$ unless $C$ is collapsed by the morphism in which case $C \cdot D=0$. Thus $D \cdot Z \geq 0$ for any positive $Z$ and hence $\langle b, a\rangle \geq 0$ for any $a$ in $K^{+}$, so $b$ is pseudoample. Conversely assume that the class of $D$ is pseudoample, where $D^{2}>0$. Write $D=$ $D_{0}+D_{\mathrm{I}}+D_{\mathrm{II}}$ where $D_{0}$ is as in Proposition 5 and $D_{\mathrm{I}}$ and $D_{\mathrm{II}}$ are divisors whose supports are on the fixed components of type I and II. We must have $D_{\mathrm{I}}=0$, for otherwise $D \cdot D_{\mathrm{I}}=D_{\mathrm{I}} \cdot D_{\mathrm{I}}<0$ and so some conic of type I must have negative intersection with $D$, contradicting the fact that $\langle b, a\rangle \geq 0$ for all $a$ in $K^{+}$. So all the connected fixed components are of type II. To show that such components do not exist (except $F_{0}$ ), we look first at the case where $D_{0}$ is irreducible. Let $E$ be the part of $D_{\text {II }}$ concentrated on some connected component $F$. Then $E \cdot D_{0}>0$, and so $E \cdot D=E D_{0}+E^{2}=\left(E \cdot D_{0}+E^{2}\right)-E \cdot D_{0}<E^{2}+2 E \cdot D_{0}$. However $E^{2}$ $+2 E \cdot D_{0}=\left(D_{0}+E_{0}\right)^{2}-D_{0}^{2}$, which equal $-2 h^{1}\left(D_{0}+E\right)$ since $l\left(D_{0}\right)=$ $l\left(D_{0}+E\right)$ and $h^{1}\left(D_{0}\right)=0$. Thus $D \cdot E$ is $<0$, and hence $D \cdot C<0$ for some irreducible component $C$ of $F$ : this contradicts the pseudoampleness of the class of $D$. It remains to consider the case where $D_{0}=F_{0}+E_{1}$ $+\cdots+E_{k}$, the $E_{j}$ elliptic curves in a pencil. Since $F_{0}$ is the only component of type II, $D=D_{0}+\sum n_{i} C_{i}$ where $F_{0}=\sum C_{i}$, and $C_{i} \cdot E_{j}=$ $1, C_{i} \cdot E_{j}=0$ for $i \geq 2$. Now if $n_{1}=1$ we can argue just as in the case 
of $D_{0}$ irreducible, and we see, in fact, that we must have $F_{0}=C_{1}$ irreducible in this case. If $n_{1} \geq 2$ then $2 C_{1}$ is a fixed divisor in $12 C_{1}+$ $E_{1}+\cdots+E_{k}$, so $l\left(2 C_{1}+E_{1}+\cdots+E_{k}\right)=l\left(C_{1}+E_{1}+\cdots+E_{k}\right)$. The Riemann-Roch theorem then gives $\left(2 C_{1}+E_{1}+\cdots+E_{k}\right)^{2}+2 h^{1}\left(2 C_{1}\right.$ $\left.+E_{1}+\cdots+E_{k}\right)=\left(C_{1}+E_{1}+\cdots+E_{k}\right)^{2}$ which gives $h^{1}\left(2 C_{1}+E_{1}+\cdots\right.$ $\left.+E_{k}\right)=3-2 k$, which is impossible since $k \geq 2$.

COROLlaRY. If $D$ is pseudoample then $h^{1}(D)=0$. (This suggests that pseudoampleness might have some significence on surfaces in generalizations of the vanishing theorem.)

Scholium. Let $\mathscr{L}$ be an invertible sheaf with $c_{1}(\mathscr{L})^{2}=d>0$ and with a pseudoample class. Then $\varphi_{\mathscr{L}}: V \rightarrow \boldsymbol{P}\left(H^{0}(V, L)\right)=\boldsymbol{P}^{g}(2 g-2=d)$ is a rational morphism of $V$ onto either

general case i) a surface of degree $2 g-2$ with rational double points

8 , and $\varphi_{\mathscr{S}}$ is birational.

hyperelliptic case ii) a rational surface of degree $g-1$, and $\varphi_{\mathscr{L}}$ is a

double covering

elliptic case iii) the $g$-fold Veronese embedding of $P^{1}$.

The image of $\varphi_{\mathscr{L}^{2}}$ is always a rational surface, or a birational image with rational double points of $V$. Finally $\varphi_{\mathscr{c} \text { s }}$ is a birational map of $V$ onto a surface with rational double points of degree $9 d$ in $(9 d+2) / 2$ dimensional projective space. If the class of $\mathscr{L}$ is ample, then $\varphi_{\mathscr{L} 3}$ is a projective embedding.

\section{§ 6. The transcendental moduli of $\mathrm{K}-3$ surfaces}

We shall review Kodaira's theory. Let $V$ be a K-3 surface. Then $L(V)=H^{2}(V, Z)$ is a free abelian group of rank 22, on which the cup product induces a quadratic form denoted by $\langle$,$\rangle (extending the previous$ usage) which is unimodular and of index -19 . Furthermore this form is even, i.e. $\langle a, a\rangle \equiv 0 \bmod 2$ for all $\alpha$ in $L(V)$. It is known then that there is some isometric isomorphism of $L(V)$ with $Z^{22}$ provided with a standard quadratic form $\langle,\rangle_{0}$, which we fix, for example by the matrix $\left(\begin{array}{llll}A & & & 0 \\ & A & & \\ & A & \\ & & B & \\ 0 & & & B\end{array}\right)=M$ where $A=\left(\begin{array}{ll}0 & 1 \\ 1 & 0\end{array}\right)$ (hyperbolic plane) and $B$ is a well known definite unimodular matrix $\left(E_{8}\right)$ with even diagonal coefficients 
which may be found in [14]. An element a of $L(V)$ is called primitive if it cannot be expressed as $n b$, with $b \in L(V), n$ an integer, unless $n= \pm 1$. It follows from a general theorem of Eichler ([6] p. 60) that if $a$ and $b$ are primitive and $\langle a, a\rangle=\langle b, b\rangle$ then there is an automorphism of the quadratic space $L(V)$ taking $a$ to $b$. By a marking $\alpha$ of $V$ we mean an isometry of $Z^{22}$ with $L(V)$ (for the given quadratic forms), giving a bases $\alpha_{1}, \cdots, \alpha_{22}$ of $L(V)$.

Since $V$ is regular the spectral sequence $H^{\cdot}(\Omega \cdot) \Rightarrow H \cdot(C)$ of de Rham cohomology degenerates so $V$ is homologically kaehlerian. Thus $H^{2}(V, \boldsymbol{R})$ $=L(V) \otimes R$ has a type decomposition into $H^{\prime}+H^{(1,1)}$ where $H^{(1,1)}$ is the space of classes of real $(1,1)$ forms and $H^{\prime}$ is the space of classes of imaginary parts of holomorphic 2-forms : in fact $H^{0}(V, K) \rightarrow H^{\prime}$ by $\varphi \rightarrow$ $\operatorname{Im}(\varphi)$ gives an isomorphism of real vector spaces, from which $H^{\prime}$ inherits a complex structure. If $\Theta$ is the sheaf of holomorphic vectors on $V$ one checks via Serre duality that $H^{2}(V, \Theta)=0$ while $H^{1}(V, \Theta)=\operatorname{Hom}_{C}\left(H^{0}(V, \Theta)\right.$, $H^{1}\left(V, \Omega^{\prime}\right)$ ) which is canonically isomorphic to $\operatorname{Hom}_{R}\left(H^{\prime}, H^{(1,1)}\right)$.

Let $S$ denote the set of oriented 2-dimensional subspace $E$ of $\boldsymbol{R}^{22}$ on which the quadratic form $\langle,\rangle_{0}$ (inherited from $Z^{22}$ ) is positive definite. $\mathrm{S}$ is a real symmetric space which can be given the structure of a complex manifold as follows: $S$ can be identified with its embedding in $\boldsymbol{P}^{21}$ by $E \rightarrow \zeta=\left(\zeta_{1}: \cdots: \zeta_{22}\right)$ where $\zeta_{i}=\zeta_{i}+\sqrt{-1} \eta_{i}, \xi=\left(\xi_{1}, \cdots, \xi_{22}\right)$ and $\eta=\left(\eta_{1}, \cdots, \eta_{22}\right)$ being an oriented orthonormal basis for $\langle,\rangle_{0}$ restricted to $E . \quad S$ is then the open subset of the quadric hypersurface $\langle\zeta, \zeta\rangle_{0}=0$ given by the condition $\langle\zeta, \bar{\zeta}\rangle_{0}>0$. Note that $S$ is not hermitian symmetric: also the group of units $G_{0}$ of the form $\langle,\rangle_{0}$ operates on $S$ in an obvious manner, but not in a properly discontinuous fashion (Siegel [15]).

Now let $v \in Z^{22}$ be a primitive vector (for the form $\langle,\rangle_{0}$. Let $S^{(v)}$ $=\left\{\zeta\right.$ in $S$ with $\left.\langle\zeta, v\rangle_{0}\right\}=0$. Equivalently, $S^{(v)}$ is the set of planes $E$ whose orthogonal complement contains $v$. If $\langle v, v\rangle_{0}=\langle w, w\rangle_{0}$ then $S^{(v)}$ and $S^{(w)}$ are conjugate by the units $G_{0}$. Furthermore if $\langle v, v\rangle>0$ then $S^{(v)}$ is an hermitian symmetric space of type IV (or type BDI, cf. Helgason: Differential Geometry and Symmetric Spaces, p. 354). If $\langle v, v\rangle_{0}>0$ then the isotropy group $G_{0}^{(v)}$ of units at $v$ acts property discontinuously on $S^{(v)}$ and the quotient $V^{(v)}$ is a 19 dimensional normal

Note: $S^{(v)}$ is nonsingular since the quadric $\langle\zeta, \zeta\rangle=0$ and the hyperplane $\langle\zeta, v\rangle=0$ are transversal at $\zeta$ of $\zeta \# v$. 
analytic space which, by a theorem of Borel-Baily can be embedded as a Zariski open set in a projective variety. Let $S^{(v) \prime}$ be the complement in $S^{(v)}$ of the union of all $S^{(v)} \cap S^{(w)}$ where $w$ runs over all primitive vectors not $=$ to $\pm v$. By the Baire category theorem $S^{(v) \prime}$ is dense in $S^{(v)}$, and the complement of a countable union of complex submanifolds. Let $W^{(v) \prime}$ be its image in $W^{(v)}$. If we let $S_{d}$ be the union of all $S^{(v)}$ with $\langle v, v\rangle_{0}=d$ then the quotient of $S_{d}$ under $G_{0}$, which we call $W_{d}$ is canonically isomorphic with any of the $W^{(v)}$. In the same way define $S_{d}^{\prime}$ and $W_{d}^{\prime}$. An important lemma of Tjurina [14] states that: for any even $d, S_{d}^{\prime}$ is dense in $S$.

Now Let $(V, \alpha)$ be a marked K-3 surface. Under the isomorphism $\alpha \otimes 1_{\boldsymbol{R}}$ of $\boldsymbol{R}^{22}$ with $H^{2}(V, \boldsymbol{R}) H^{\prime}$ corresponds to one of our space $E$, with an orientation given by the complex structure which $H^{\prime}$ carries. This gives a point $\lambda(V, \alpha)$ in $S$ : if its homogenous coordinates are $\zeta_{1}, \cdots, \zeta_{22}$ this means that $\zeta_{1} \alpha_{1}+, \ldots,+\zeta_{22} \alpha_{22}$ is the class of a holomorphic 2-form on $V$. Note that the Picard number of $V=$ the rank of the lattice contained in the orthogonal complement of the space $E$ corresponding to $\lambda(V, \alpha)$. The fundamental theorem of Andreotti-Weil-Kodaira (see [9]) states that if $\left(V_{t}, \alpha_{t}\right)$ is a locally complete family of deformations of $(V, \alpha)$ then the map $t \rightarrow \lambda\left(V_{t}, \alpha_{t}\right)$ is a locally biholomorphic map of the parameter space onto an open subset of $S$. It follows that the set $U$ of $\zeta$ in $S$ of the form $\lambda(V, \alpha)$ is open in $S$ : it is also clearly $G_{0}$ invariant. On the other hand $U$ is also connected: for by the Lemma of Tjurina mentioned above, to prove this it suffices to prove that $U \cap S_{d}^{\prime}$ is connected for some $d$. But if $\lambda(V, \alpha) \in S_{d}^{\prime} V$ is a generic $\mathrm{K}-3$ and can be characterized by our Proposition 3. Kodaira proved the irreducibility of the family of all such $V$ for $d=0$, and, as we have remarked, for $d=2,4,6,8$, this is also easy to prove. Thus $U$ is a connected open set in $S$.

\section{REFERENCES}

[1] M. Artin, Some numerical criteria for contractability of curves on algebraic surfaces. Amer. J. Math 84 485-496 (1962).

[2] M. Artin, On isolated rational singularities of surfaces. Amer. J. Math. 88 129-136 (1966).

[3] E. Brieskorn, Über die auflösung gewisser singularitäten von holomorphen abbildungen. Math. Annalen 166 76-102 (1966).

[4] E. Brieskorn, Rationale singularitäten komplexer flächen. Inventiones Math. 4 336-358 (1968). 
[5] B. d'Orgevel, Sur les surfaces algébriques dont tous les genres. sont 1. Paris, Gauthier-Villars (1945).

(6 ] M. Eichler, Quadratische formen und orthogonal gruppen. Springer Verlag (1952).

[ 7 ] F. Enriques, Le superficie algebriche Bologna. (1949).

[8] K. Kodaira, On compact analytic surfaces I. Annals of Math. 71 111-152 (1960).

[9] K. Kodaira, On the structure of compact complex analytic surfaces I. Amer. J. Math. 86 751-798 (1964).

[10] T. Matsusaka, On some analytic familities of polarized algebraic varieties. J. Math. Kyoto Univ. 5 no. 3 279-312 (1966).

[11] J. Milnor, On simply connected 4-manifolds. Int. Symp. Alg. Top. Mexico City 19.

[12] D. Mumford, The topology of normal singularities. Publ. I.H.E.S. no. 9 (1961).

[13] J. Nakai, A criterion of an ample sheaf. Amer. J. Math. 85 14-26 (1963).

[14] K. Safarevic et al., Algebraic surfaces. A.M.S. translation, Providence (1967).

[15] C. Siegel, Discontinuous groups, Gesammelte Abhandlungen II.

[16] J.-P. Serre, Faisceaux algébriques cohérent. Annals of Math. 61 197-278 (1955).

[17] O. Zariski, Complete linear systems on normal varieties. Annals of Math. 55 552-592 (1952).

Brandeis University 